\title{
PROCESSO dE CRIAÇÃO dA COMISSÃO de SAÚDE DE LONDRINA E REGIÃO, ESTADO DO PARANÁ, BRASIL
}

\author{
Process of creation of a region Health Commission in \\ the city of Londrina, state of Paraná, Brazil
}

Reilly Alberto Aranda Lopes ${ }^{1}$, Felipe Assan Remondi ${ }^{2}$, Pablo Guilherme Caldarelli ${ }^{3}$

1. Diretor Geral do Hospital Dr. Anísio Figueiredo (Zona Norte de Londrina) - Secretaria de Estado da Saúde do Paraná (SESA). Londrina-PR. ORCID: http://orcid.org/0000-0002-1648-8405

2. Farmacêutico da $17^{a}$ Regional de Saúde - Secretaria de Estado da Saúde do Paraná (SESA). LondrinaPR. ORCID: http://orcid.org/0000-0003-3213-1366

3. Professor do Departamento de Medicina Oral e Odontologia Infantil- Universidade Estadual de Londrina (UEL). Londrina-PR. ORCID: http://orcid.org/0000-0002-4589-9713

CONTATO: Reilly Alberto Aranda Lopes | Endereço: Rua Odilon Braga, 199 | Cj. Sebastião de Melo Cesar | CEP 86084-600 | Londrina-PR | Telefone: (43) 3376-4600 | E-mail: contato@ reillylopes.com

COMO CITAR: Lopes RAA, Remondi FA, Caldarelli PG. Processo de criação da Comissão de Saúde de Londrina e região, Estado do Paraná, Brasil. R. Saúde Públ. 2019 Jul;2(1):113-124

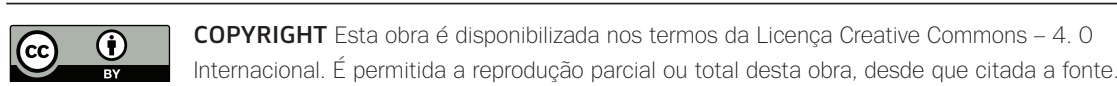

RESUMO Este artigo descreve a ação de um grupo de gestores em saúde Londrina-PR e região que se reuniu com o objetivo de fortalecer a integração e abordagem sistêmica para os problemas experimentados no Sistema Único de Saúde (SUS). Trata-se de um relato de experiência, estruturado por meio de uma abordagem críticoreflexiva, descrevendo as fases e ferramentas utilizadas no projeto, que ao longo de 2018 resultou na conformação de 5 (cinco) eixos de atuação: Apostar na Atenção Primária à Saúde; Fortalecer a produção assistencial na média e alta complexidade; Qualificar a capacidade instalada da atenção hospitalar; Aprimorar a integração, regulação e comunicação no cuidado e Garantir o financiamento e alocação eficiente de recursos. A partir destes caminhos, o grupo busca construir uma unidade entorno de uma agenda comum, com soluções inovadoras de médio e longo prazo. 
PALAVRAS-ChAVE: Gestor de Saúde. Política de Saúde. Saúde Pública. Problemas Sociais. Política Nacional de Promoção a Saúde. Financiamento Governamental.

ABSTRACT This article describes the action of a group of health managers in the city of Londrina, state of Paraná, and in the region, who met with the objective of enhancing the integration and systemic approach to problems in the Unified Health System (SUS). This is an experience report, structured through a critical-reflexive approach, describing the phases and tools used in the project in 2018 that resulted in the creation of five axes of action: Invest on Primary Health Care; Strengthen care production in medium and high complexity services; Qualify the installed capacity of hospital assistance; Improve integration, regulation and communication in health care; and Ensure funding and efficient allocation of resources. The group seeks a common agenda to act on the identified axes, with innovative medium- and long-term strategies.

KEYWORDS: Health Manager. Health Policy. Public Health. Social Problems. National Policy for Health Promotion. Govern Financing.

\section{INTRODUÇÃO}

$\mathbf{A}$ s políticas de saúde têm sido alvo de atenção e tema de grandes discussões no Brasil nos últimos 30 anos. Segundo a Constituição Federal', a saúde é um direito do cidadão e o Estado tem o dever de assegurála mediante políticas específicas, obedecendo à igualdade de acesso e possibilitando a utilização dos serviços conforme a necessidade dos usuários.

Entretanto, o acesso à saúde nem sempre ocorre no momento da necessidade. Apesar de mais de três décadas de implementação do Sistema Único de Saúde (SUS), o país ainda experimenta dificuldades na concretização do direito à saúde que perpassam questões de financiamento, organização do modelo de atenção e dificuldades em garantir o acesso em tempo oportuno à população². Nesse contexto, ordenar este atendimento para garantir a eficiência e sustentabilidade do sistema consiste em um desafio para a gestão de saúde.

Em todo o país, a procura pelos serviços de saúde cresce ano após ano com tendência ascendente $^{2}$ em razão do aumento populacional, transição epidemiológica e demográfica e crise econômica. Da pressão decorrente deste crescimento e as limitações enfrentadas pelo poder público em garantir a expansão do sistema ${ }^{3}$ advém um cenário de crise e a necessidade de busca de novos caminhos para a gestão. 
Nesta perspectiva, o Conselho Nacional de Secretários de Saúde (CONASS) ${ }^{4}$ afirma que é necessário ofertar e dar acesso à população a serviços organizados em redes de atenção à saúde, com adequado processo de regionalização e hierarquização para ganhos de escala e materialização da integralidade da atenção. O movimento de construção desta rede assistencial perpassa a ruptura com o modelo de gestão tradicional, burocratizado, hierárquico, fragmentado e com relações de poder nem sempre harmônicas.

Cabe aos gestores, portanto, ampliarem seu horizonte de atuação, superando os muros das instituições ou dos hospitais em que trabalham para maior integração junto à comunidade e os diversos atores que compõem a rede de atenção ${ }^{5}$. bem como a busca por tecnologias inovadoras e a construção do pensamento sistêmico.

Reproduzindo o cenário nacional, os desafios vivenciados na organização SUS em Londrina e sua região, Estado do Paraná, levou um grupo de gestores - líderes do setor de saúde pública - iniciarem um processo de articulação de um espaço colaborativo para análise dos problemas experimentados, suas causas e consequências, de forma a construir caminhos convergentes e integrados entre as instituições envolvidas para superar a fragmentação da gestão e suas consequentes soluções pontuais, que têm seus impactos limitados sob a ótica do sistema de saúde como um todo.

Com o objetivo de conformar uma agenda única a ser divulgada e debatida com atores estratégicos para permitir melhorias consistentes do atendimento aos usuários, ao longo de 2018 o grupo de trabalho identificou e sistematizou os problemas de Londrina e região, a partir dos quais iniciou-se o processo de divulgação e construção da unidade necessária para a criação de planos de ação para melhoria do cenário em curto, médio e longo prazo.

O presente trabalho busca relatar a experiência da Comissão de Saúde de Londrina e Região, a metodologia aplicada e os resultados alcançados ao longo de 2018.

\section{METODOLOGIA}

Trata-se de um relato de experiência, estruturado por meio de uma abordagem críticoreflexiva e descritiva, sobre processo de criação da Comissão de Saúde de Londrina-PR e região, no qual se encontram descritas sua concepção, as metodologias utilizadas e os principais resultados alcançados ao longo de 2018.

Para além do registro dos trabalhos e sua divulgação com o intuito de ampliação dos debates e replicação em outras localidades, o presente relato foi elaborado como produto do curso de Especialização em Gestão Hospitalar do Programa de Apoio e Qualificação de Hospitais Públicos e Filantrópicos do SUS Paraná (HOSPSUS), da Secretaria de Estado da Saúde do Paraná (SESA). A missão do HOSPSUS é qualificar a resposta hospitalar em todas as regiões de saúde do Paraná contribuindo para o desenvolvimento de hospitais sociais e sanitariamente essenciais para atender as necessidades da população do Estado.

\section{RELATO DE EXPERIÊNCIA E DISCUSSÃO}

Sabe-se que os recursos, individuais ou coletivos, são sempre escassos para fazer frente às despesas provenientes de um ou mais tratamentos de saúde. No contexto do sistema público de saúde, é preciso que os gestores tenham presente a importância de equacionar as necessidades da população com os recursos disponíveis, estruturando políticas de saúde capazes de beneficiar o maior número possível de pessoas. Nesse sentido, quando se trata de administrar bem os recursos disponíveis, é indispensável que estes sejam usados de forma eficaz, efetiva e eficiente ${ }^{5}$. 
Londrina é um município situado ao norte do Estado do Paraná, com 553.393 habitantes ${ }^{6}$ e constituindo-se como referência direta para serviços de saúde da $17^{a}$ Regional de Saúde do Paraná, composta pelos municípios: Alvorada do Sul, Assaí, Bela Vista do Paraíso, Cafeara, Cambé, Centenário do Sul, Florestópolis, Guaraci, Ibiporã, Jaguapitã, Jataizinho, Londrina, Lupionópolis, Miraselva, Pitangueiras, Porecatu, Prado Ferreira, Primeiro de Maio, Rolândia, Sertanópolis e Tamarana, que totalizam uma população de 943.434 habitantes ${ }^{6}$. Além disso, para situações de maior complexidade é referência para toda a macrorregião norte do Estado do Paraná, perfazendo 1,8 milhão de habitantes?

Neste cenário, os desafios enfrentados pelo SUS impactam diretamente em Londrina e municípios adscritos, constituindo ambiente de elevada complexidade gerencial, cujos problemas agudos e crônicos são percebidos e manifestados pela população por meio de um quadro comum: hospitais e prontos-socorros superlotados, filas para consultas e procedimentos especializados, infraestrutura deficitária, entre outros ${ }^{8}$. Para Bittencourt (2009) ${ }^{9}$, a superlotação, principal efígie das dificuldades do sistema, é definida como uma saturação do limite operacional de um serviço, com pacientes nos corredores por causa da falta de leitos disponíveis, o não recebimento de ambulâncias por conta de saturação operacional, salas de espera para consulta médica lotada, equipes de profissionais no limite da exaustão e mais de uma hora de espera para o atendimento médico.

A pressão advinda desta descrição simplista da realidade leva a população e os gestores, em maior ou menor grau de intensidade, a uma compreensão limitada e consequente busca por soluções pontuais para problemas de elevada complexidade e característica sistêmica. Em que pese a importância e a expressiva ampliação da capacidade instalada do SUS nos últimos 30 anos, uma mobilização apenas em torno do aumento da oferta de pronto-atendimentos, consultas e procedimentos especializados, ainda que importantes, se constituem como soluções inócuas em médio ou longo prazo ${ }^{10}$.

Como forma de ilustrar, um estudo apresentado em um hospital de grande porte da cidade de São Paulo, por exemplo, entre 50\% e 70\% dos pacientes que procuram o serviço são classificados como "urgência relativa", sendo que muitos deles poderiam aguardar alguns dias por uma consulta eletiva sem maiores riscos ${ }^{11}$. Essa realidade não é exclusividade daquele hospital e revela que soluções pontuais nem sempre constituem como melhor alternativa para os problemas emergentes do sistema, uma vez que a alocação adequada dos recursos escassos em unidades de atenção básica traria ganhos de efetividade e eficiência para o sistema como um todo.

Segundo Campos (2018) $)^{12}$ a ampliação e consolidação do SUS requer dos diversos atores sociais a mobilização, articulação e pensamento sistêmico, que parte dos problemas agudos e cotidianos para identificar suas causas estruturantes e propor alternativas consistentes no campo das políticas públicas:

\footnotetext{
"O SUS encontra-se bastante fragmentado, que torna precária a governança do sistema na lógica de redes de atenção integral. É fundamental que seja ampliada a integração sistêmica do SUS" (Campos, 2018, p. 1711) ${ }^{12}$.
}

Partindo desta inquietação e da compreensão que a intervenção sobre as situações pontuais experimentadas individualmente pelas diversas instituições carece de uma articulação entre os gestores locais, iniciou-se uma mobilização democrática e de caráter aberto, com a incorporação de novos atores e olhares a cada reunião.

A proposta do grupo baseou-se no Princípio de Pareto, que afirma que existe um forte 
desequilíbrio entre causas e efeitos, entre esforços e resultados e entre ações e objetivos alcançados, e que, de uma maneira genérica, $80 \%$ dos resultados que obtemos estão relacionados com 20\% dos nossos esforços, ou seja, uma minoria de ações leva a maior parte dos resultados ${ }^{13}$.

Então, se os resultados e objetivos alcançados estão relacionados às ações executadas, o grupo buscou identificar os principais problemas (20\%) que, se solucionados, representariam uma melhoria significativa (80\%) nos serviços de saúde da região. Assim, ao convergir atores para análise da situação, sistematização dos problemas e identificação daqueles com maior grau de relevância constituiuse o primeiro passo para uma ação coordenada que tem em seu horizonte o médio e longo prazo.

Para que o trabalho fosse capaz de gerar uma unidade entre os atores e um coeficiente colaborativo, optou-se por realizar um trabalho participativo, para construção de uma pauta única, onde as diferentes expressões de ideias foram apresentadas e sugestões expostas.

Neste sentido, foram convidados a participar inicialmente das discussões os representantes dos hospitais públicos e privados de Londrina, representantes da Secretaria de Saúde do Estado e do Município, e um parlamentar de Londrina. A proposta do grupo era iniciar um trabalho de discussão com a inclusão de novos atores a partir das discussões sistematizadas e capazes de mobilizá-los em torno de um produto concreto, ainda que não terminado.

As primeiras reuniões tiveram sua pauta aberta, adotando a metodologia de Brainstorm ou como é conhecido, "Tempestade de Ideias". Essa técnica foi desenvolvida pelo publicitário norte-americano Alex Osborn, em 1949, e é capaz de identificar as possiveis causas do problema investigado, uma maneira disciplinada de geração de novas ideias a partir de discussões em grupo ${ }^{14}$.

Em cada reunião, um relator registrou os principais itens da discussão de forma clara e objetiva, submetendo o registro para aprovação do grupo. Para tornar o esforço de sistematização mais claro e acessível, sempre que possível o registro das reuniões foi convertido em representações visuais, com uma ferramenta chamada Wordcloud, que permite criar uma "nuvem de palavras" utilizando formas e imagens de modo que se permita uma melhor visualização do conteúdo proposto, dando destaques àqueles termos que mais vezes foram citados. Com as palavras em destaque, pode-se observar uma "visão situacional" dos problemas de saúde da região, que permitiram uma análise inicial dos principais fatores que interferem no cenário (Figura 1).

A partir da percepção dos envolvidos, um subgrupo de sistematização organizou os problemas, discutindo a relação entre eles, com o agrupamento e indicação de relações que foram visualmente representadas em uma árvore explicativa (Figura 2). A projeção visual e as sucessivas discussões para a validação dos produtos intermediários permitiram o amadurecimento das discussões, integração de novos atores e compreensão sistêmica dos problemas.

Ao final de quatro reuniões ao longo de 2018, a árvore explicativa foi composta por três eixos principais (temas), 11 ramos (nós) e 31 problemas, cada qual com um conjunto de elementos que representam como os diversos atores percebem esta situação (Quadro 1).

Todos os problemas identificados já foram em maior ou menor grau objeto de análises no plano nacional. Contudo, o exercício de problematização e sistematização deles, a partir da realidade local, foram de fundamental importância para a construção do significado e da unidade dos atores em torno de uma agenda unificada.

Tendo o processo de problematização atingido a saturação das discussões, os mesmos foram priorizados para permitir a construção 
Figura 1 Tecnologias de síntese e representação visual dos problemas discutidos: Wordcloud das discussões da $2^{\underline{a}}$ reunião.

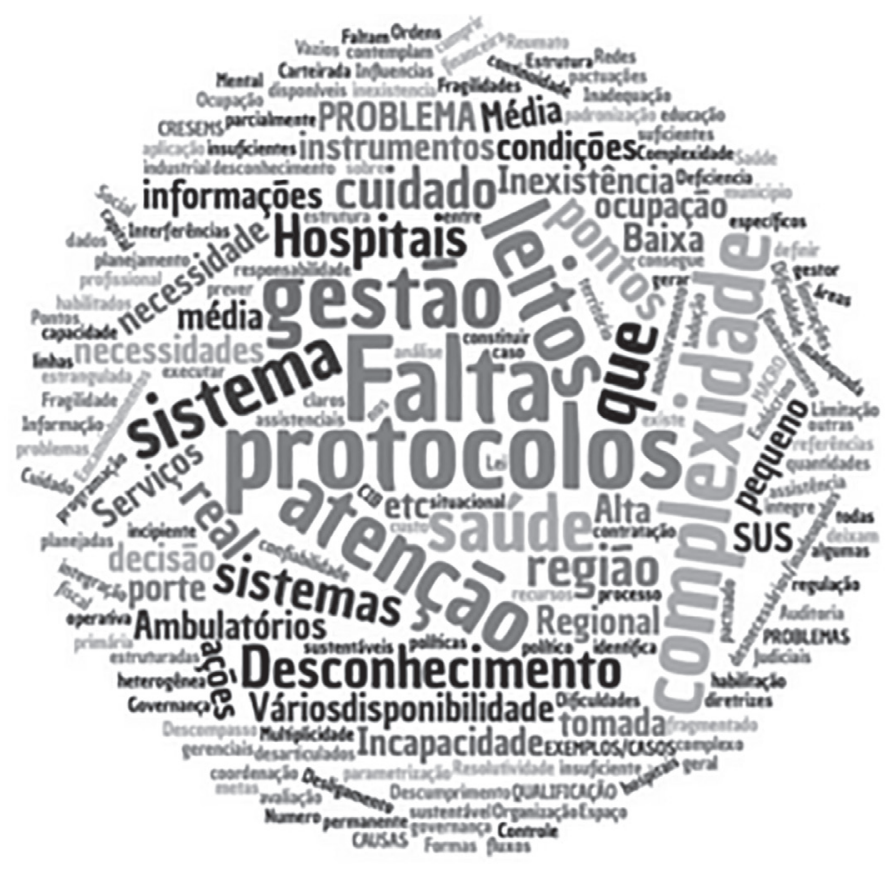

Fonte: Elaborado pelos autores (2018).

Figura 2 Tecnologias de síntese e representação visual dos problemas discutidos: Árvore explicativa dos problemas, suas evidências e interrelações.

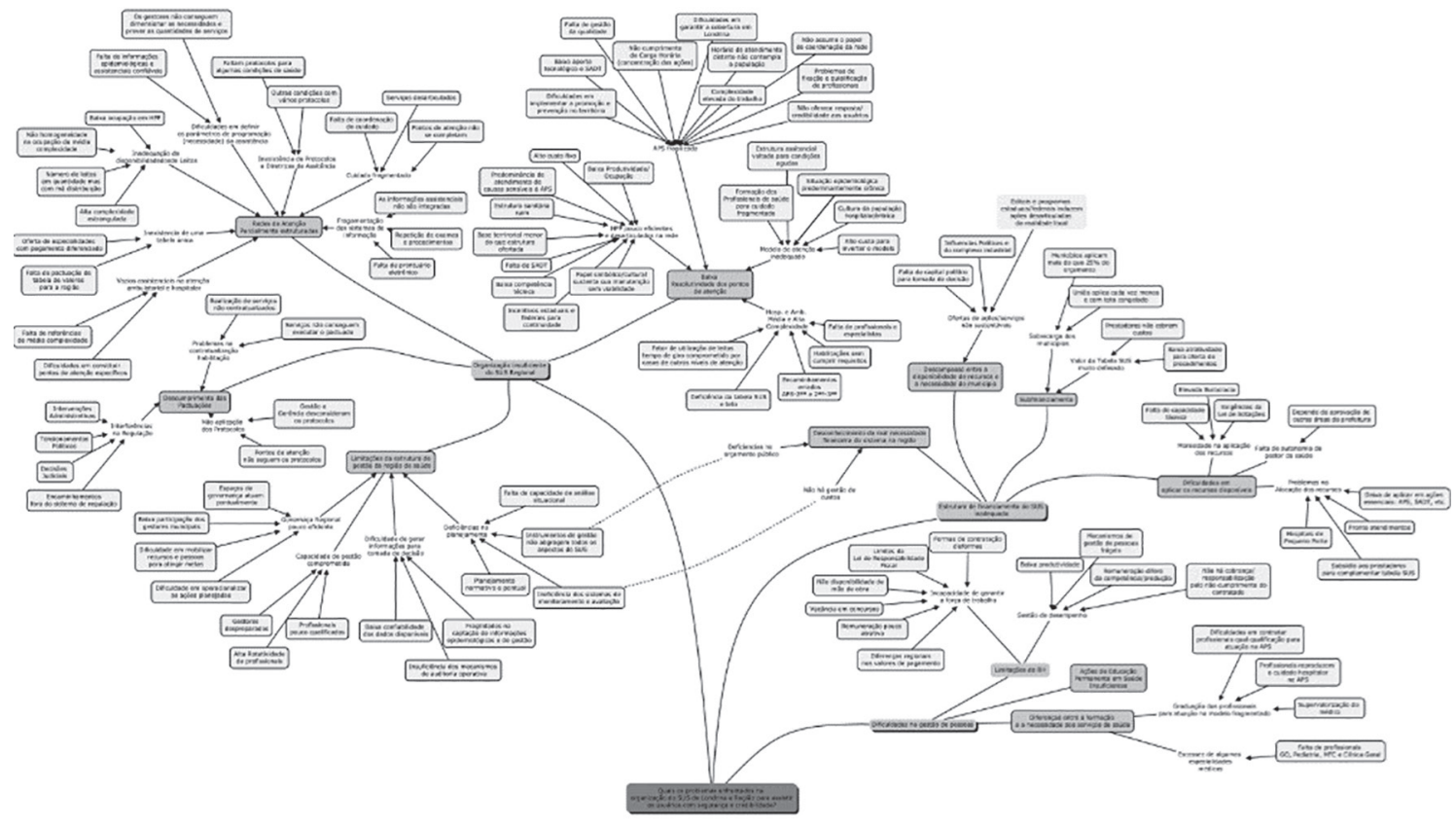

Para visualização da árvore completa e detalhada acesse: http://bit.ly/arvoreproblemas

Fonte: Elaborado pelos autores (2018). 
Quadro 1 Problemas percebidos pelo grupo, ordenados conforme a pontuação obtida por meio da matriz GUT.

\begin{tabular}{|c|c|}
\hline $\mathbf{N}^{\circ}$ & PROBLEMA \\
\hline 1 & Atenção Primária Fragilizada \\
\hline 2 & Hospitais de Pequeno Porte pouco eficientes e desarticulados na rede \\
\hline 3 & Inadequação da disponibilidade de Leitos \\
\hline 4 & Sobrecarga dos municípios \\
\hline 5 & Valor da Tabela SUS muito defasado \\
\hline 6 & Hosp. e Amb. Média e Alta Complexidade \\
\hline 7 & Problemas na contratualização e habilitação \\
\hline 8 & Problemas na Alocação dos recursos \\
\hline 9 & Interferências na Regulação \\
\hline 10 & Cuidado fragmentado \\
\hline 11 & Capacidade de gestão comprometida \\
\hline 12 & Incapacidade de Garantir a força de Trabalho \\
\hline 13 & Governança Regional pouco eficiente \\
\hline 14 & Escassez de algumas especialidades médicas \\
\hline 15 & Não aplicação dos protocolos \\
\hline 16 & Ofertas de ações/serviços não sustentáveis \\
\hline 17 & Vazios assistenciais na atenção ambulatorial e hospitalar \\
\hline 18 & Fragmentação dos sistemas de informação \\
\hline 19 & Ações de Educação Permanente em Saúde Insuficientes \\
\hline 20 & Deficiências no orçamento público \\
\hline 21 & Inexistência de uma tabela única \\
\hline 22 & Dificuldade de gerar informações para tomada de decisão \\
\hline 23 & Dificuldades em definir os parâmetros de programação (necessidade) da assistência \\
\hline 24 & Gestão de Desempenho \\
\hline 25 & Modelo de atenção inadequado \\
\hline 26 & Não há gestão de custos \\
\hline 27 & Deficiências no planejamento \\
\hline 28 & Morosidade na aplicação dos recursos \\
\hline 29 & Inexistência de Protocolos e Diretrizes de Assistência \\
\hline 30 & Falta de autonomia do gestor da saúde \\
\hline 31 & Graduação dos profissionais para atuação no modelo fragmentado \\
\hline
\end{tabular}


assertiva de ações para aquilo com maior impacto sobre a organização dos serviços, conforme prediz o Princípio de Pareto ${ }^{13}$. As duas reuniões seguintes foram destinadas à priorização dos problemas e aprovação do relatório final dos trabalhos.

Para isto foi construído um formulário online, onde cada participante classificou cada um dos problemas elencados, levando em conta os parâmetros da Matriz GUT como forma de reduzir a subjetividade da avaliação entre os pares. A matriz tem esse nome pelo fato de levar em consideração a Gravidade, a Urgência e a Tendência de cada problema ${ }^{15}$. Enquanto a Gravidade representa o impacto do problema analisado caso ele venha a acontecer, a Urgência diz respeito ao tempo disponível ou necessário para resolver um determinado problema analisado, e a Tendência avalia o potencial de crescimento do problema e a probabilidade do problema se tornar maior com o passar do tempo.

Baseado nesta proposta, cada participante atribuiu notas de 1 a 5 para os parâmetros indicados e o conjunto de respostas foi totalizado para classificar em ordem decrescente os problemas a serem abordados na melhoria do processo (Quadro 1). Este tipo de análise permitiu estabelecer a ordem de prioridade dos problemas, auxiliando na estratégia de trabalho da comissão ${ }^{15}$.

Para melhor compreensão dos problemaschave e caminhos a serem explorados foram construídos cinco eixos de atuação que contemplaram especialmente os problemas priorizados. Na ocasião das discussões, ainda sem ter a perspectiva de definir ações para cada problema, os eixos figuraram como caminhos para gerar uma ação proativa e convergente, inclusive com a ampliação das discussões para outros atores-chave.

Para cada eixo, o subgrupo de sistematização elaborou um breve texto de apresentação que procurou registrar as discussões do grupo e o contexto no qual se inserem, agregando elementos teóricos que sustentam ou apontam caminhos a serem seguidos. Tal qual todos os produtos, o texto foi discutido e validado pelo grupo. Os 5 (cinco) pilares identificados pela comissão foram:

\section{Apostar na Atenção Primária à Saúde:}

A Atenção Primária à Saúde (APS) consiste na estratégia de organização dos serviços de saúde com base na oferta das ações em territórios definidos, onde são desenvolvidas ações de promoção, prevenção e recuperação da saúde de maneira multidisciplinar, com estímulo à participação social e da educação em saúde ${ }^{16}$. Dados oficiais do Ministério da Saúde para o ano de 2015 apontam que na Regional de Saúde de Londrina, em média 21\% de todos os atendimentos hospitalares ocorreram por causas sensiveis à APS, sendo que em unidades de pronto atendimento ou hospitais de menor porte/ complexidade esta proporção é ainda maior. Isso é um dos inúmeros exemplos que apontam para os efeitos sistêmicos de uma APS fragilizada e. não por acaso, foram exaustivamente registrados pelo grupo em outros problemas. Apostar na APS, com a construção de soluções para garantia da cobertura, pronta disponibilidade de todos os profissionais, qualificação da produção do cuidado em Redes de Atenção à Saúde, não se trata apenas de uma decisão ordinária, mas significa apostar na melhoria dos níveis de saúde da população com o uso eficiente e sustentável dos recursos disponíveis. No âmbito regional temos dois cenários que requerem atenção: municípios de maior porte que têm dificuldades na garantia da cobertura, mas apresentam corpo técnico qualificado e bons resultados; e municípios de menor porte com cobertura para a totalidade da população, mas que requerem ações para qualificação do alcance de suas ações.

\section{Fortalecer as redes de atenção e produção assistencial na média e alta complexidade: A atenção especializada é marcada por diferentes gargalos, principalmente no que se refere ao acesso. A dificuldade para a garantia de acesso a serviços especializados decorre do modelo de atenção adotado, da resolutividade da APS e}


também do dimensionamento e organização da oferta dos serviços ${ }^{17}$. As discussões da comissão apontam para a necessidade, antes mesmo de discutir a ampliação ou não deste conjunto de serviços, da busca por sua integração em uma rede de atenção e um modelo de atenção integrado, com forte vinculação à APS. Ainda, no contexto regional, muitos municípios estabeleceram sistemas autônomos sem a devida articulação regional para consultas e exames especializados que apontam para a necessidade do fortalecimento da regionalização. Os pontos-chave para compreensão e intervenção sobre o problema foram:

a. Os vazios assistenciais na atenção ambulatorial e hospitalar;

b. O modelo de atenção inadequado, com o cuidado fragmentado;

c. As dificuldades em definir os parâmetros de programação das necessidades;

d. Os problemas na contratualização e habilitação de prestadores;

e. As limitações da estrutura de gestão da região de saúde.

III. Qualificar a capacidade instalada da atenção hospitalar: Definir o número de leitos hospitalares necessários para atender uma dada população é uma tarefa complexa, pois envolve analisar não apenas a oferta desses serviços, mas também a sua demanda e características locais, o que determina o apontamento de um índice ideal bastante temerário, apesar de teorias e recomendações sobre o tema. Considerando a recomendação do Ministério da Saúde (2015) ${ }^{18}$ e população de 2012 estima-se que a 17a Regional de Saúde deva possuir 1.639 leitos gerais e 176 leitos de Unidade de Terapia Intensiva (UTI) para seus usuários. Atualmente contamos com 2.022 leitos gerais e 130 leitos de UTI. Para além de um quantitativo próximo das necessidades é preciso considerar que a $17^{\text {a }}$ Regional de Saúde é referência também para a Macrorregião Norte, bem como a distribuição e resolutividade dos leitos disponíveis.
Ao fazê-lo, a comissão foi unânime em apontar uma situação desigual, onde os principais desafios se referem à:

a. Inadequação da disponibilidade de leitos:

i. Baixa ocupação em Hospitais de Pequeno Porte (HPP);

ii. Não homogeneidade na ocupação de unidades de média complexidade;

iii. Alta complexidade estrangulada.

b. HPPs pouco eficientes e deserticulados na rede;

c. Deficiências nos Hospitais e Ambulatórios de Média e Alta Complexidade, como valores insuficientes da tabela SUS, falta de profissionais e especialistas, habilitações para serviços sem cumprir os requisitos mínimos e encaminhamentos de casos que eram passíveis de manejo em outros níveis assistenciais.

Assim, é preciso, portanto, trilhar o caminho no fortalecimento da regionalização para garantia do acesso sem perda da economia de escala, com reorganização da disponibilidade de leitos hospitalares para permitir o redirecionamento dos recursos para onde ele possibilitará benefícios em maior escala, como na Atenção Primária, no fortalecimento dos serviços hospitalares que continuariam habilitados e na ampliação da atenção ambulatorial especializada.

IV. Aprimorar a integração, regulação e comunicação no cuidado: Apesar dos inúmeros esforços e avanços observados em anos recentes, é preciso intensificar a conformação de Redes de Atenção para atendimento a condições agudas e crônicas com resolutividade, oportunidade e eficiência ${ }^{19}$. Pensar em um sistema de saúde articulado em rede pressupõe a integração dos diversos pontos de atenção para produção de uma ação proativa, contínua, com cuidado longitudinal e tendo a APS como ordenadora deste processo, sendo que dois novos elementos devem ser agregados à discussão: a regulação do cuidado e a comunicação entre os pontos de atenção. Ao 
se pensar na integração da rede e nos processos regulatórios, os problemas/fatores identificados pela Comissão que carecem atenção são:

a. Interferências na regulação, advindas de tensionamentos políticos, decisões judiciais e encaminhamentos fora do sistema de regulação;

b. Cuidado fragmentado em função da falta de coordenação do cuidado, serviços desarticulados e pontos de atenção que não se completam;

c. Não aplicação ou inexistências de protocolos e diretrizes assistenciais

\section{Garantir o financiamento e alocação} eficiente de recursos: Entre os grandes desafios do SUS destacam-se o financiamento e a alocação dos recursos disponíveis de forma eficiente. Não se trata de atribuir os problemas vivenciados apenas à falta de recursos, uma vez que os quatro primeiros caminhos se dedicam somente à necessidade de ganhos de eficiência, mas não será possível mobilizar as transformações necessárias ou a sustentabilidade do sistema sem discutir a disponibilidade dos recursos e a alocação dos mesmos ${ }^{20}$. Diante deste cenário e na perspectiva da Comissão é preciso uma mobilização para ampliação da base de financiamento do sistema e, paralelamente, garantir que os recursos captados sejam alocados buscando maior eficiência nos sistemas de saúde, com apontamentos das seguintes situações:

a. Sobrecarga de financiamento dos municípios em detrimento da União;

b. Incapacidade de garantir a força de trabalho no SUS;

c. Oferta de ações/serviços não sustentáveis;

d. HPP pouco eficientes e desarticulados na rede;

e. Valor da tabela SUS muito defasado;

f. Inexistência de uma tabela unificada para contratação de serviços; g. Morosidade na aplicação dos recursos;

h. Problemas na alocação dos recursos em ações desarticuladas das prioridades regionais;

i. Falta de autonomia do gestor da saúde para definição da alocação dos recursos.

Como foi dito, mais do que identificar problemas desconhecidos o processo de problematização com os atores partiu da realidade de Londrina e região, e foi fundamental para gerar a unidade e convergência necessárias para que a Comissão construa as alternativas inovadoras para enfrentamento dos problemas.

Tendo o relatório definido e divulgado, a Comissão passou a realizar apresentações públicas nas instituições que compuseram as discussões e. sempre que possível, buscou novos espaços e atores para divulgação da agenda proposta. O relatório foi apresentado para as equipes do Hospital Evangélico de Londrina, Hospital Zona Sul de Londrina, Hospital Zona Norte de Londrina, 17 ${ }^{\mathrm{a}}$ Regional de Saúde de Londrina, Hospital Universitário de Londrina, Associação Médica de Londrina, Grupo Sallus (Saúde Londrina União Setorial), Cismepar (Consórcio Intermunicipal de Saúde do Médio Paranapanema), Comissão Intergestores Bipartite Regional e Comitê Executivo da Saúde, composto por representantes do poder executivo, judiciário e ministério público, abrangendo mais de 200 pessoas. Destas apresentações, foram acolhidas novas adesões ao texto base a proposta de mobilização.

É preciso, no entanto, transbordar o discurso para além do setor saúde com o foco em entidades capazes de gerar uma mobilização social, como a Câmara de Vereadores de Londrina, Associação de Municípios do Médio Paranapanema, Associação Comercial e Industrial de Londrina (ACIL), Sociedade Rural do Paraná (SRP), deputados estaduais e federais da região e outros, buscando envolver a sociedade civil organizada no engajamento do projeto. 


\section{CONSIDERAÇÕES FINAIS}

Ao longo de todo processo ficou evidente a complexidade dos problemas vivenciados $\mathrm{e}$ a necessidade de ações coordenadas entre os gestores, prestadores, profissionais de saúde, universidades, políticos e sociedade civil organizada. Muitas situações decorrem de problemas cuja governabilidade direta não esteja no nível local, mas que dependem dos atores que aqui produzem suas atividades para propor medidas capazes de contorná-los.

Com este trabalho pôde-se perceber que a fragmentação da gestão tem sido um problema constante na região, sendo este talvez o principal avanço da proposta de mobilização: constituir um espaço supra-institucional, colaborativo e capaz de acolher e aprofundar a discussão dos problemas dos diversos atores para reforçar a necessidade do pensamento e atuação sistêmicos.

Por se tratar de uma experiência exitosa, os caminhos adotados podem propiciar que outras regiões do Estado busquem a articulação descrita como forma de garantir melhorias de médio e longo prazo, e o fortalecimento do SUS em sua totalidade.

Para o ano de 2019, os principais desafios a serem superados estão relacionados a continuidade da mobilização dos atores da região, a difusão das discussões para além do setor saúde, a mobilização dos representantes políticos de Londrina e região e a construção de espaços que sejam capazes de propor ações concretas para os caminhos descritos.

\section{AGRADECIMENTOS}

Às instituições e seus diversos representantes que contribuíram até o momento com a mobilização proposta (em ordem alfabética): $17^{\mathrm{a}}$ Regional de Saúde, Associação Médica de Londrina, Comissão Intergestores Bipartite da $17^{a}$ RS. Conselho Municipal de Saúde de Londrina, Consórcio Intermunicipal do Médio Paranapanema, Gabinete do Deputado Estadual Tiago Amaral, Grupo SALUS - Saúde Londrina União Setorial, Hospital Cristo Rei de Ibiporã, Hospital de Olhos de Londrina, Hospital do Câncer de Londrina, Hospital Dr. Anísio Figueiredo, Hospital Dr. Eulalino Ignácio de Andrade, Hospital Evangélico de Londrina, Hospital São Rafael de Rolândia, Hospital Universitário Regional do Norte do Paraná, Irmandade Santa Casa de Londrina, Santa Casa de Cambé e Secretaria Municipal de Saúde de Londrina.

\section{CONFLITOS DE INTERESSE}

Os autores declaram inexistir conflitos de interesse com o relato apresentado.

\section{REFERÊNCIAS}

1. Brasil. Constituição da República Federativa do Brasil, de 05 de outubro de 1988. Brasília (DF): Senado Federal; 1988.

2. Santos NR. SUS 30 anos: o início, a caminhada e o rumo. Ciênc saúde coletiva [Internet]. 2018. [citado 2019 Mar 05] ; 23(6):1729-36. Disponivel em: http://www.scielo.br/scielo. php?script=sci_arttext\&pid=S1413-81232018000601729\&lng=pt.

3. Azambuja CRC. Importância das medidas de gestão no controle da superlotação hospitalar. [Internet] [monografia]. Santa Maria (RS): Universidade Federal de Santa Maria; 2014. [citado 2019 Fev 15]. Disponivel em: https://repositorio.ufsm.br/handle/1/11730.

4. Brasil, Conselho Nacional de Secretários de Saúde (CONASS). Propostas do CONASS aos candidatos à Presidência da República. [homepage na Internet]. [citado 2019 Fev 15]. Disponível em: http://www.conass.org.br/propostas-do-conass-aos-candidatospresidencia-da-republica/.

5. Burmester H. Gestão em Saúde. Rev. Adm. Saúde. [Internet] 2017 [citado 2019 Fev 15];17(66): editorial. Disponivel em: http:// cqh.org.br/ojs-2.4.8/index.php/ras/article/view/5/16.

6. Secretaria de Estado da Saúde do Paraná (PR). Plano Diretor de Regionalização. [internet]. Paraná: Secretaria de Estado da Saúde: 2015. [citado 2019 Fev 15]. Disponível em: http://www.saude. pr.gov.br/arquivos/File/00_-_NDS/PDR_2015.pdf

7. Ministério Público do Estado do Paraná. Regionais de saúde. [homepage na Internet]. [citado 2019 Fev 15]. Disponivel em: http://www.saude.mppr.mp.br/modules/conteudo/conteudo. php?conteudo=522 
8. Santos CAS, Santo EE. Análise das causas e consequências da superlotação dos serviços de emergências hospitalares: uma revisão bibliográfica. Revista Saúde e Desenvolvimento. [Internet]. 2014 [citado 2019 Fev 15];5(3):31-44. Disponivel em: https://www. uninter.com/revistasaude/index.php/saudeDesenvolvimento/article/view/187/210.

9. Bittencourt RJ, Hortale VA. Intervenções para solucionar a superlotação nos serviços de emergência hospitalar: uma revisão sistemática. Cad Saúde Pública [Internet]. 2009 [citado 2019 Fev 15]:25(7):1439-1454. Disponivel em: http:// www.scielo.br/scielo.php?script=sci_arttext\&pid=S0102$311 \times 2009000700002 \&$ Ing=en.

10. Viacava F, Oliveira RAD, Carvalho CC, Laguardia J, Bellido JG. SUS: oferta, acesso e utilização de serviços de saúde nos últimos 30 anos. Ciênc. saúde coletiva [Internet]. 2018 Jun [citado 2019 Fev 17]:23(6):1751-1762. Disponivel em: http://www.scielo.br/scielo. php?script=sci_arttext $\&$ pid=S1413-81232018000601751\&lng=en.

11. Hospital Sírio Libanês. Pronto atendimento ou consulta: como fazer a escolha certa. [homepage na Internet]. [citado $2019 \mathrm{Fev}$ 15]. Disponivel em: https://www.hospitalsiriolibanes.org.br/suasaude/Paginas/pronto-atendimento-consulta-como-fazer-escolha-certa.aspx.

12. Campos GWS. SUS: o que e como fazer?. Ciênc. saúde coletiva [Internet]. 2018 Jun [citado 2019 Fev17]; 23(6):1707-14. Disponível em: http://www.scielo.br/scielo.php?script=sci_arttext\&pid=S1413$81232018000601707 \&$ lng=en.

13. Brophy P. Coulling K. Quality management for information and library managers. Brookfield: Aslib Gower, 1996.

14. Barreto, RM. Criatividade em propaganda. 12. ed. São Paulo: Summus, 2004.

15. Daychoum, M. 40+4 ferramentas e técnicas de gerenciamento. 3. ed. Rio de Janeiro: Brasport, 2010.

16. Mendonça MHM, Matta GC, Gondim R, Giovanella L, organizadores. Atenção primária à saúde no Brasil: conceitos, práticas e pesquisa. Rio de Janeiro: Fiocruz: 2018.

17. Silva CR, Carvalho BG, Cordoni Júnior L, Nunes EFPA. Difficulties in accessing services that are of medium complexity in small municipalities: a case study. Ciênc. saúde coletiva [Internet]. 2017 [citado 2019 Fev 15]:22(4):1109-20. Disponivel em:

http://www.scielo.br/scielo.php?script=sci_arttext\&pid=S141381232017002401109\&lng=pt.

18. Brasil. Ministério da Saúde. Portaria de Consolidação $n^{\circ} 01$. Consolidação, portaria normativa, direito à saúde, usuário do SUS, organização e administração, funcionamento, Sistema Único de Saúde (SUS). Diário Oficial da União, 03 out 2017; Suplemento: 1.

19. Brasil. Conselho Nacional de Secretários de Saúde (CONASS). A atenção primária e as redes de atenção à saúde. Brasilia: CONASS, 2015. 127 p.

20. Brasil, Conselho Nacional de Secretários Municipais de Saúde, Conselho Nacional de Secretários de Saúde. Nota Conjunta - CONASEMS/CONASS Financiamento da Saúde [Internet]. Brasília: CONASEMS; 2017 [citado 2019 Fev 16]. Disponivel em: http://www. conasems.org.br/wp-content/uploads/2017/09/Nota-ConjuntaConass-e-Conasems-Financiamento-e-Gest\%C3\%A30-25.09_final.pdf. 